\title{
A GENERALIZATION OF HASSE'S SERIES REPRESENTATION FOR THE RIEMANN ZETA FUNCTION
}

\author{
SUMIT KUMAR JHA
}

Abstract. We derive the following generalization of Hasse's series representation for the Riemann zeta function

$$
\zeta(s)=\frac{(-1)^{n}}{s-1} \sum_{k=0}^{\infty} \frac{1}{k+1} \sum_{a=0}^{k}(-1)^{a} \cdot S(n, a) \cdot a ! \cdot \sum_{m=0}^{k-a}\left(\begin{array}{c}
k \\
m
\end{array}\right) \cdot \frac{(-1)^{m}}{(m+1)^{s+n-1}}
$$

where $s \neq 1, n$ being any non-negative integer, and $S(n, k)$ denotes the Stirling number of the second kind. The special cases of the above representation are Ser's and Hasse's global series representation for the Riemann zeta function.

Let $\zeta(s)$ represent the Riemann zeta function. H. Hasse [1] proved that

$$
\zeta(s)=\frac{1}{s-1} \sum_{n=0}^{\infty} \frac{1}{n+1} \sum_{k=0}^{n}\left(\begin{array}{l}
n \\
k
\end{array}\right) \frac{(-1)^{k}}{(k+1)^{s-1}} \quad(\text { where } s \neq 1) .
$$

Joseph Ser [2] proved that

$$
\zeta(s)=\frac{1}{s-1} \sum_{n=0}^{\infty} \frac{1}{n+2} \sum_{k=0}^{n}\left(\begin{array}{l}
n \\
k
\end{array}\right) \frac{(-1)^{k}}{(k+1)^{s}} \quad(\text { where } s \neq 1) .
$$

We prove the following generalization of the above two results.

Theorem 1. Let $\zeta(s)$ represent the Riemann zeta function. Then we have

$$
\zeta(s)=\frac{(-1)^{n}}{s-1} \sum_{k=0}^{\infty} \frac{1}{k+1} \sum_{a=0}^{k}(-1)^{a} \cdot S(n, a) \cdot a ! \cdot \sum_{m=0}^{k-a}\left(\begin{array}{c}
k \\
m
\end{array}\right) \cdot \frac{(-1)^{m}}{(m+1)^{s+n-1}}
$$

where $s \neq 1, n$ being any non-negative integer, and $S(n, k)$ denotes the Stirling number of the second kind.

Letting $n=0$ and $n=1$ in the above representation gives us Hasse's representation (1) and Ser's representation (2), respectively.

2010 Mathematics Subject Classification. $11 \mathrm{M} 06$.

Key words and phrases. Riemann zeta function, Hasse's representation, Global series representation, Ser's representation. 
Proof. We start with the following integral [3]

$$
(\alpha+\beta) \zeta(\alpha+\beta+1)=\int_{0}^{\infty} \frac{\mathbf{L i}_{\alpha}(-1 / t) \mathbf{L} \mathbf{i}_{\beta}(-t)}{t} d t \quad(\alpha+\beta \neq 0),
$$

where $\zeta(\cdot)$ is the Riemann zeta function, and $\mathbf{L} \mathbf{i}_{\beta}(-t)$ is the polylogarithm function.

Letting $\alpha=-n$, a non-negative integer, and $\beta=s$, a complex number, in equation (3) we have

$$
(s-n) \cdot \zeta(s-n+1)=\int_{0}^{\infty} \frac{\mathbf{L} \mathbf{i}_{-n}(-1 / t) \mathbf{L} \mathbf{i}_{s}(-t)}{t} d t .
$$

We will use the following representation [4]

$$
\mathbf{L i}_{-n}(-1 / t)=(-1)^{n-1} \frac{1}{t} \sum_{k=0}^{n} k ! S(n, k)\left(\frac{t}{1+t}\right)^{k+1}(-1)^{k} .
$$

The polylogarithm function, $\mathbf{L i}_{s}(z)$, has the following series representation $[5$, Theorem 2.1]

$$
\mathbf{L i}_{s}(z)=\sum_{k=0}^{\infty}\left(\frac{-z}{1-z}\right)^{k+1} \sum_{m=0}^{k}\left(\begin{array}{c}
k \\
m
\end{array}\right) \frac{(-1)^{m+1}}{(m+1)^{s}},
$$

for all $s, z \in \mathbb{C}$ with $\Re(z)<1 / 2$. These representations allow us to evaluate right side of equation (4) as

$$
\begin{array}{r}
\int_{0}^{\infty} \frac{\mathbf{L i}_{-n}(-1 / t) \mathbf{L i}_{s}(-t)}{t} d t \\
=(-1)^{n-1} \sum_{k=0}^{\infty} \int_{0}^{\infty} \frac{t^{k}}{(1+t)^{k+2}} d t \sum_{a+b=k}(-1)^{a} \cdot S(n, a) \cdot a ! \cdot \sum_{m=0}^{b}\left(\begin{array}{c}
k \\
m
\end{array}\right) \cdot \frac{(-1)^{m+1}}{(m+1)^{s}} \\
=(-1)^{n-1} \sum_{k=0}^{\infty} \frac{1}{k+1} \sum_{a+b=k}(-1)^{a} \cdot S(n, a) \cdot a ! \cdot \sum_{m=0}^{b}\left(\begin{array}{c}
k \\
m
\end{array}\right) \cdot \frac{(-1)^{m+1}}{(m+1)^{s}} \\
=(-1)^{n-1} \sum_{k=0}^{\infty} \frac{1}{k+1} \sum_{a=0}^{k}(-1)^{a} \cdot S(n, a) \cdot a ! \cdot \sum_{m=0}^{k-a}\left(\begin{array}{c}
k \\
m
\end{array}\right) \cdot \frac{(-1)^{m+1}}{(m+1)^{s}} .
\end{array}
$$

\section{REFERENCES}

1. Hasse, Helmut (1930). Ein Summierungsverfahren fr die Riemannsche $\zeta$-Reihe [A summation method for the Riemann $\zeta$ series]. Mathematische Zeitschrift (in German). 32 (1): 458-464. doi:10.1007/BF01194645 
2. Ser, Joseph (1926). Sur une expression de la fonction $\zeta(s)$ de Riemann [Upon an expression for Riemann's $\zeta$ function]. Comptes rendus hebdomadaires des sances de l'Acadmie des Sciences (in French). 182: 10751077.

3. Handbook of Mellin Transforms by Yu. Brychkov, O. Marichev, N, Savischenko, 2019.

4. Stirling Numbers and Polylogarithms by Steven E. Landsburg. Link: http://www.landsburg.com/query.pdf

5. J. Guillera, J. Sondow, Double integrals and infinite products for some classical constants via analytic continuations of Lerchs transcendent, J. Ramanujan J (2008) 16: 247. https://doi.org/10.1007/s11139-0079102-0

International Institute of Information Technology, Hyderabad, India

E-mail address: kumarjha.sumit@research.iiit.ac.in 